\title{
The Overexpression of Epidermal Growth Factor (EGF) in HaCaT Cells Promotes the Proliferation, Migration, Invasion and Transdifferentiation to Epidermal Stem Cell Immunophenotyping of Adipose-Derived Stem Cells (ADSCs)
}

\author{
Yueping Mao ${ }^{1, *}$, Jianchi $\mathrm{Ma}^{1, *}$, Yue $\mathrm{Xia}^{2}$, Xiaoyuan $\mathrm{Xie}^{2}$ \\ ${ }^{1}$ Department of Dermatology, Sun Yat-sen Memorial Hospital, Sun Yat-sen University, Guangzhou, China \\ ${ }^{2}$ Department of Dermatology, The Third Affiliated Hospital, Sun Yat-sen University, Guangzhou, China
}

\begin{abstract}
Background and Objectives: The application of adipose derived stem cells (ADSCs) in skin repair has attracted much attention nowadays. Epidermal growth factor (EGF) participates in the progress of skin proliferation, differentiation and so forth. We aimed to explore the role of EGF in the proliferation, invasion, migration and transdifferentiation into epidermal cell phenotypes of ADSCs.

Methods and Results: ADSCs were extracted from adipose tissues from patient. Immunophenotyping was determined by flow cytometry. Overexpressed EGF or siEGF was transfected by lentiviruses. EGF was determined by enzyme linked immunosorbent assay (ELISA) or western blot. ADSCs and HaCaT cells were co-cultured by Transwell chambers. Conditioned medium (CM) was obtained from cultured $\mathrm{HaCaT}$ cells and used for the culturing of ADSCs. Cell viability was tested by 3-(4,5-dimethylthiazol-2-yl)-2,5-diphenyltetrazolium bromide (MTT) assay. Invasion rate was measured by Transwell invasion assay and migration rate by wound healing test. mRNA and protein levels were measured by qPCR and western blot respectively. The extracted cells from adipose tissues were identified as ADSCs by morphology and immunophenotyping. The expression of EGF was up or down regulated constantly in $\mathrm{HaCaT}$ cell line after transfection. EGF overexpression upregulated the proliferation, migration and invasion rates of ADSCs, and EGF expression regulated the expression of cytokeratin-19 (CK19) and integrin- $\beta$ as well.

Conclusions: EGF could be served as a stimulus to promote the proliferation, migration, and invasion as well as the transdifferentiation into epidermal stem cell immunophenotyping of ADSCs. The results showed that EGF had a promising effect on the repair of skin wound.
\end{abstract}

Keywords: EGF, ADSCs, Repair, Transdifferentiation, Tissue engineering

Received: December 26, 2018, Revised: May 28, 2019,

Accepted: May 28, 2019, Published online: February 29, 2020

Correspondence to Xiaoyuan Xie

Department of Dermatology, The Third Affiliated Hospital, Sun

Yat-sen University, No. 600 Tianhe Road, Guangzhou 510630, China

Tel: +86-020-85253333, Fax: +86-020-85253336

E-mail: xiaoyuanx_xiexy@163.com

*These authors contributed equally to this work.

(c) This is an open-access article distributed under the terms of the Creative Commons Attribution Non-Commercial License (http://creativecommons.org/ licenses/by-nc/4.0/), which permits unrestricted non-commercial use, distribution, and reproduction in any medium, provided the original work is properly cited.

Copyright (c) 2020 by the Korean Society for Stem Cell Research

\section{Introduction}

Severe skin tissue loss can be caused by trauma, extensive burns, diabetes or other chronic diseases (1). Currently, autologous, allogeneic skin grafts and artificial substitutes are commonly used in clinical practice, however, these methods have limited effects $(1,2)$, as problems such as new traumas in the donor area, limited donor sources and immune rejection still exist $(1,2)$. Therefore, skin wound repair remains an urgent issue to be solved $(1,2)$.

Recently, with the continuous development of stem cell 
technology, the application of mesenchymal stem cells in skin repair has attracted much attention (3). Adipose-derived stem cells (ADSCs) can be obtained from the human body through minimally invasive methods or from the excess adipose tissue in the body $(4,5)$. The source of ADSCs is abundant, and the culturing of ADSCs is easy in vitro $(4,5)$. The amount of ADSCs in the same tissue is much larger than that in the bone marrow mesenchymal stem cells, and ADSCs, which have good self-proliferation and multi-lineage differentiation potentials, can differentiate into tissue cells such as adipocytes, osteoblasts, hepatocytes and endothelial cells (4, 5). Brzoska et al. (6) reported that all-trans retinoic acid (ATRA) induced the differentiation of adipose-derived stem cells into epidermal cells, indicating that adipose-derived stem cells have the ability to differentiate into epidermal cells across the germ layer. Yao et al. (7) revealed that the transplantation of ADSCs could promote the healing of skin deep partial-thickness scald wound of rabbit. Thus, the usage of ADSCs in the repair of wound skin shows a promising effect.

Epidermal growth factor (EGF) is a type of polypeptide, which is composed of 53 amino acids and promoting mitosis (8). After hydrolysis, it exerts its biological activity on and participates in the progress of skin proliferation, differentiation, apoptosis and carcinogenesis (9). Previous studies had shown that EGF could induce the early development of teeth and eyelids in mice inhibit the secretion of gastric acid, as well as promote the growth of epidermis and the keratinization process (8). In addition, researchers also reported that ADSCs treated with EGF by three-dimensional culturing method would differentiate to an epithelial phenotype $(10,11)$. Therefore, further study of EGF in the tissue engineering has a scientific significance.

In view of above evidence, we aimed to explore the role of EGF produced by $\mathrm{HaCaT}$ cells in the proliferation, invasion, migration and transdifferentiation of ADSCs into epidermal cell phenotypes. Our study provides supporting evidences for ADSCs to be used as ideal seed cells in tissue engineering.

\section{Materials and Methods}

\section{The extraction of ADSCs and cell culturing}

The protocol of this study was approved by the ethics board of Sun Yat-sen Memorial Hospital, Sun Yat-sen University (approval number: SY2017010745). Adipose tissue was collected from a female patient who aged 29 years old. The patient signed the informed consents before the study has been conducted. Under sterile conditions, about
$10 \mathrm{~g}$ of the remaining abdominal subcutaneous adipose tissue of the patient who took the plastic surgery was sent to the laboratory within $1 \mathrm{~h}$. The tissue was first immersed in PBS (Solarbio Life Sciences, Beijing, China) containing penicillin $(300 \mathrm{U} / \mathrm{ml})$ and streptomycin $(300 \mu \mathrm{g} / \mathrm{ml})$ (V900929-100 ml, Sigma, Shanghai, China) in a super clean bench for $10 \mathrm{~min}$ and then rinsed twice with $2 \times$ PBS for $5 \mathrm{~min}$. Blood vessels and connective tissues were carefully removed using ophthalmic scissors, and the adipose tissue was cut into $1 \mathrm{~mm} \times 1 \mathrm{~mm} \times 1 \mathrm{~mm}$ size. The tissues were gathered in a flask and added with $10 \mathrm{ml}$ of 0.1\% type I collagenase (SCR103, Sigma, Shanghai, China), and they were vigorously shaken and digested in a water bath at $37^{\circ} \mathrm{C}$ for $1 \mathrm{~h} .10 \%$ FBS $(10 \mathrm{ml})$ (10099141, Gibco, Shanghai, China) was added to neutralize collagenase, and the samples were centrifuged at $1500 \mathrm{round} / \mathrm{min}$ for 10 min in a high-speed centrifuge at low temperature. The upper adipose tissue and supernatant were discarded, and the samples were resuspended with DMEM (10569044, Thermo Fisher, Waltham, USA) medium and sieved, and further centrifuged at $1500 \mathrm{round} / \mathrm{min}$ for $10 \mathrm{~min}$. Next, the supernatant was discarded, and the samples were resuspended with DMEM medium and then placed in a 25 $\mathrm{cm}^{2}$ cell culture flask and incubated with $5 \% \mathrm{CO}_{2}$ at $37^{\circ} \mathrm{C}$. The medium was exchanged after $24 \mathrm{~h}$ to remove residual red blood cells and unattached cells. Meanwhile, HaCaT cell line was obtained from the Type Culture Collection of the Chinese Academy of Sciences, Shanghai, China and cultured in DMEM Medium supplied with 10\% FBS and $5 \% \mathrm{CO}_{2}$ at $37^{\circ} \mathrm{C}$. Medium was renewed every $2 \sim 3$ days. The cells were subcultured at a ratio of $1: 3$ when they reached the logarithmic growth phase by being digested with $0.125 \%$ trypsin-EDTA (25300054, Gibco, Shanghai, China) at $37^{\circ} \mathrm{C}$. The cells were observed under a microscope (Olympus IX71, Tokyo, Japan) when cell density reached $80 \% \sim 90 \%$ of culture flask.

\section{Immunophenotyping by flow cytometry}

$100 \mu 1$ of $5 \times 10^{7} / \mathrm{ml}$ single cell suspension with $20 \mu 1$ of fluorescently labeled mouse anti-human CD13 (CD13PE, 560998, BD, Shanghai, China, 1 : 500), CD14 (CD14APC, 561708, BD, Shanghai, China, 1:500), CD34 (CD34-PerCP, 340430, BD, Shanghai, China, $1: 500$ ), CD44 (CD44-APC, 559942, BD, Shanghai, China, 1 : 500), CD90 (CD90-FITC, 561969, BD, Shanghai, China, 1 : 500), and CD105 (CD105-PE, 560839, BD, Shanghai, China, $1: 500$ ) antibodies were added to a clean plastic tube and incubated at $4^{\circ} \mathrm{C}$. The cells were then added with $2 \mathrm{ml} \mathrm{PBS}$, mixed and centrifuged at $1500 \mathrm{round} / \mathrm{min}$ for $5 \mathrm{~min}$. Next, the supernatant was discarded and $200 \mu \mathrm{l}$ 
PBS was added to each tube and well mixed. The tubes were loaded on flow cytometry and tested. Data was analyzed by Cell-Quest software.

\section{Lentivirus, transfection}

cDNAs encoding EGF, siEGF and negative control sequence (nonsense) were cloned into the lentiviral expression vectors pLenti6/CMV/V5-DEST (synthesized by Genepharma, Shanghai, China). The sequences of the EGF siRNA were 5'-AAUCCUUAUGAGGAG UCGA-3' and 5'-CCACCACUAUUCCGUAAGA-3'. HaCaT cells were centrifuged at $1000 \mathrm{round} / \mathrm{min}$ for $5 \mathrm{~min}$, resuspended by trypsin and then collected. $5 \times 10^{5}$ cells were added into $1 \mathrm{ml}$ of the culture medium, resuspended and counted, and then transferred to a 24 -well plate. The cells were further added a volume of virus solution at multiplicity of infection (MOI) of 40 with $500 \mu 1$ of culture medium. Ploybrene transfection enhancer (No.107689, Sigma, Shanghai, China) at a ratio of $1: 200$ was added, and the cells were then incubated in an incubator. After $24 \mathrm{~h}$, the virus-containing medium was removed, and fresh culture medium was added to continue the culturing. $\mathrm{HaCaT}$ cells were grouped as control, Mock, EGF, NC and siEGF groups. Control group was a blank control, and Mock, EGF, NC, and siEGF were treated with lentiviral vector, lentiviral vector with $\mathrm{EGF}$, negative control lentiviral vector of siEGF and lentiviral vector with siEGF respectively.

\section{Determination of EGF by enzyme linked immunosorbent assay (ELISA)}

After the transfection and culturing of $\mathrm{HaCaT}$ cells for $24 \mathrm{~h}, 48 \mathrm{~h}$ and $72 \mathrm{~h}$ respectively, the co-culturing of ADSCs and HaCaT cells for 14 days and the culturing of ADSCs with conditioned medium (CM) from modified $\mathrm{HaCaT}$ cells, the determination of EGF was then conducted according to the instructions given (ab100504, Abcam, Shanghai, China). Briefly, the serum-free supernatants of the cells were collected and $100 \mu 1$ of the samples were added to a 96-well reaction plate. The plate was thoroughly mixed with the samples and incubated at $37^{\circ} \mathrm{C}$ for $120 \mathrm{~min}$. Thereafter, the plate was washed with washing liquid for $4 \sim 6$ times and dried on a filter paper, and then $100 \mu 1$ of working solution with primary antibody was added to each well, and the plate was incubated at $37^{\circ} \mathrm{C}$ for $60 \mathrm{~min}$. The plate was then washed, and $100 \mu 1$ the working solution with enzyme-labeled antibody was added to each well, and the plate was incubated at $37^{\circ} \mathrm{C}$ for $60 \mathrm{~min} .100 \mu \mathrm{l}$ working solution with substrate was added to each well after the plate was washed, and then the plate was placed in a dark drawer at $37^{\circ} \mathrm{C}$ for $15 \mathrm{~min}$. $100 \mu 1$ stop solution was added to each well, and the OD value at $450 \mathrm{~nm}$ was read on a microplate reader (Multiskan, Thermo, Waltham, USA).

\section{The set of co-culturing system}

The Transwell plate (Corning, USA) was used to establish a cell co-culture system. There were different groups as follows: control group (The upper layer had no cells, while the lower layer was inoculated with $4 \times 10^{4}$ ADSCs); HaCaT-Mock group (The upper layer was inoculated with $\mathrm{HaCaT}$ cells containing lentiviral vector, while the lower layer was inoculated with $4 \times 10^{4} \mathrm{ADSCs}$ ); HaCaT-EGF group (The upper layer was inoculated with HaCaT cells containing lentiviral vector with EGF, while the lower layer was inoculated with $4 \times 10^{4}$ ADSCs); HaCaT-NC group (The upper layer was inoculated with $\mathrm{HaCaT}$ cells containing negative control lentiviral vector of siEGF, while the lower layer was inoculated with $4 \times 10^{4}$ ADSCs); HaCaT-siEGF group (The upper layer was inoculated with $\mathrm{HaCaT}$ cells containing siEGF, while the lower layer was inoculated with $4 \times 10^{4}$ ADSCs). The cells were cultured for 14 days.

\section{The culturing of ADSCs with CM}

The cells of HaCat-Mock, HaCat-EGF, HaCat-NC, HaCat-siEGF groups were collected when the cell confluence reached more than $85 \%$. The cells were then incubated in a serum-free medium for the next $48 \mathrm{~h}$, and the supernatant was gathered. After the supernatant was filtered with $0.22 \mu \mathrm{m}$ filter membrane, the cells were marked as CM of each group and stored at $-80^{\circ} \mathrm{C}$. ADSCs were cultured and grouped as follows: control group (cells with DMEM medium); Mock-CM group (cells with 50\% DMEM and 50\% CM from HaCat-Mock group); EGF-CM group (cells with 50\% DMEM and 50\% CM from HaCat-EGF group); NC-CM group (cells with 50\% DMEM and 50\% CM from HaCat-NC group); siEGF-CM group (cells with 50\% DMEM and 50\% CM from HaCatsiEGF group).

\section{3-(4,5-dimethylthiazol-2-yl)-2,5-diphenyltetrazolium bromide (MTT) assay}

Cell viability was measured after ADSCs have been co-cultured with HaCaT cells for 14 days and after the ADSCs have been cultured CM from modified HaCaT cells for $24 \mathrm{~h}, 48 \mathrm{~h}$, and $72 \mathrm{~h}$ respectively. MTT Cell Proliferation Assay Kit (ab211091, Abcam, Shanghai, China) was used to measure the cell viability. The cells were gathered and loaded into a 96-well plate when cell 
density reached $5 \times 10^{6}$ cells $/ \mathrm{ml}$. Medium was discarded and $50 \mu 1$ of serum-free media and MTT Reagent were added into each well. The plate was incubated at $37^{\circ} \mathrm{C}$ for $3 \mathrm{~h}$. After the incubation, $150 \mu \mathrm{l}$ of MTT Solvent was added into each well. The plate was then wrapped in foil and shaken on an orbital shaker for 15 min. Finally, OD at $590 \mathrm{~nm}$ was read on a microplate reader (Multiskan, Thermo, Waltham, USA).

\section{Transwell invasion assay}

Invasion rate was assessed by Transwell chambers $(8 \mu \mathrm{m}$, BD Biosciences, CA, USA). Briefly, the cells were collected and loaded into the top chamber when cell density reached $2.5 \times 10^{4}$ cells $/ \mathrm{cm}^{2} .200 \mu 1$ medium with $10 \%$ FBS (10099158, Gibco, Shanghai, China) was added into bottom chamber. The chambers were incubated at $37^{\circ} \mathrm{C}$ for 48 h. Cells that did not invade through the membrane pores were removed by swab from the top chamber. The cells that did not invade into the bottom chamber were fixed and stained with $0.5 \%$ crystal violet (C6158, Sigma, Shanghai, China) for $30 \mathrm{~min}$. The cells were then counted under an inverted microscope (Olympus IX71, Tokyo, Japan) and photographed.

\section{Wound healing test}

Cells were incubated on 6-well plates until being merged into a monolayer. Thereafter, $0.5 \mathrm{~cm}$ gap was made by a $50 \mu 1$ pipette tip and the cells were cultured for $24 \mathrm{~h}$. The cells were washed with PBS for 2 times after medium has been removed and then supplied with fresh medium. The cells were cultured for the next $24 \mathrm{~h}$. The distances between the edges of the gaps were then measured. The healing of the gaps of the monolayer of the cells was photographed under an inverted microscope (Olympus IX71, Tokyo, Japan) at the same observation site.

\section{Measurement of mRNA levels by qPCR}

Total RNA was extracted by Trizol (Invitrogen, Shanghai, China) and cDNA was synthesized by iScriptTM cDNA Synthesis Kit (Bio-Rad, CA, USA) following the instructions of manufacturers. qPCR was conducted using Fast Start Universal SYBR Green Master kit (Roche, Shanghai, China). The total volume of reaction system was $20 \mu 1$ and prepared as follows: $2 \times$ SYBER Green master mix $10 \mu 1$, cDNA template $2 \mu 1$, forward primer $(10 \mu \mathrm{M}) 0.5 \mu \mathrm{l}$, reverse primer $(10 \mu \mathrm{M}) 0.5 \mu \mathrm{l}, \mathrm{ddH}_{2} \mathrm{O}$ $7 \mu$. Procedures of PCR were as follows: at $95^{\circ} \mathrm{C}$ for 30 sec, at $95^{\circ} \mathrm{C}$ for $15 \mathrm{sec}$, at $60^{\circ} \mathrm{C}$ for $30 \mathrm{sec}$, and at $72^{\circ} \mathrm{C}$ for $2 \mathrm{~min}, 40$ cycles. PCR was performed in $\mathrm{CFX} 96$
Touch $^{\text {TM }}$ (\#6093, Bio-Rad, CA, USA). Primers were listed in Table 1.

\section{Extraction and determination of proteins}

The cells were lysed by RIPA (89900, Thermo Fisher, Shanghai, China) and centrifuged at $4{ }^{\circ} \mathrm{C}, 16000 \mathrm{~g}$ for 30 min following the instructions of manufacturer. The supernatant of cells was gathered and stored at $-80^{\circ} \mathrm{C}$. The concentration of total protein was determined by the Pier$\mathrm{ce}^{\mathrm{TM}}$ BCA Protein Assay Kit (Thermo Fisher, Waltham, USA). $25 \mu 1$ samples and standard proteins were diluted to $1,0.5,0.25,0.125,0.0625 \mathrm{~g} / \mathrm{ml}$, respectively, and then loaded into the left column of a 96-well plate. $200 \mu 1$ Working Regent was added to each well and mixed with the samples or standard proteins thoroughly on a plate shaker for $30 \mathrm{sec}$. The plate was covered and incubated at $37^{\circ} \mathrm{C}$ for $30 \mathrm{~min}$. The absorbance at $562 \mathrm{~nm}$ was measured on a microplate reader (Multiskan, Thermo, Waltham, USA). The concentration of total protein was calculated by EXCEL software according to the standard curve.

\section{Western blot}

Samples were boiled at $95^{\circ} \mathrm{C}$ for $15 \mathrm{~min}$. Thereafter, proteins were separated by $10 \%$ SDS-PAGE electrophoresis at $100 \mathrm{~V}$ for $2 \mathrm{~h}$. The proteins were then transferred at $90 \mathrm{~V}$ for $1.5 \mathrm{~h}$ onto a PVDF membrane and blocked with 5\% non-fat milk for $1 \mathrm{~h}$ at RT. Primary antibody anti-EGF antibody (ab9695, Abcam, San Francisco, USA, 1 : 1000), anti-beta actin antibody (ab8227, Abcam, San Francisco, USA, 1 : 2000), anti-cytokeratin 19 antibody (ab15463, Abcam, San Francisco, USA, 1 : 1000), and anti-integrin- $\beta 1$ antibody (ab179471, Abcam, San Francisco, USA, $1: 2000)$ were incubated with the membrane in sealing bags at $4{ }^{\circ} \mathrm{C}$ overnight. The membrane was washed 3 times with PBST (Solarbio Life Sciences, Beijing, China) for 5 min each time. The secondary antibody IgG H\&L (HRP) (ab6721, Abcam, San Francisco, USA, $1: 2000$ ) was then incubated with the membrane for

Table 1. Primers used in quantitative PCR

\begin{tabular}{ll}
\hline \multicolumn{1}{c}{ Genes } & \multicolumn{1}{c}{ Primers } \\
\hline \multirow{2}{*}{ EGF } & (F) 5'-GCAGATGGGTCAATGCAAC-3' \\
& (R) 5'-GGGACAGGAGCCCTTATCA-3' \\
CK19 & (F) 5'-CTGAGTGACATGCGAAGCCAATA-3' \\
& (R) 5'-CAGTAACCTCGGACCTGCTCATC-3' \\
Integrin- $\beta$ & (F) 5'-AAGCGAAGGCATCCCTGAAAG-3' \\
$\beta$ & (R) 5'-TGCTGCATTCACAATGTCTACCAA-3' \\
& (F) 5'-CCCTGGAGAAGAGCTACGAG-3' \\
& (R) 5'-CGTACAGGTCTTTGCGGATG-3'
\end{tabular}


$1 \mathrm{~h}$ at RT. The membrane was washed 3 times in PBST for 5 min once more and detected by Pierce ${ }^{\mathrm{TM}}$ ECL plus western blotting substrate (Thermo Fisher, Waltham, USA). Blots of proteins were photographed and analyzed.

\section{Statistical analysis}

Data was analyzed by GraphPad Prism v7.0 software. One-way and two-way analysis of variance were used in multiple comparisons. $\mathrm{p}<0.05$ indicated a statistical significance.

\section{Results}

\section{ADSCs were identified by morphology and immunophenotyping}

In order to distinguish ADSCs from primary cultured cells, the third-generation cells with good growth status were observed under an inverted phase contrast microscope and their immunophenotypings were determined by flow cytometry. As shown in the images (Fig. 1A), the cells were in two forms, one was the fibroblast-like cells that had long protruding feet at both ends, another was in a flat irregular or polygonal shape with a large protrusion. In addition, the fused cells were arranged in such a directional pattern as "swirl". The outcomes of cytometry showed that CD13, CD44, CD90 and CD105 were highly expressed, while the CD14 and CD34 were lowly expressed (Fig. 1B), indicating that these cultured cells extracted from tissues could be confirmed as ADSCs.

\section{The expression of EGF was up or down regulated constantly in HaCaT cell line}

To confirm that whether EGF was successfully overexpressed or suppressed in $\mathrm{HaCaT}$ cells and whether the effectiveness could last for a period of time, we measured the expression of EGF by qPCR, western blot and ELISA. The results showed that the expression of EGF in EGF group was much higher than that in mock group, and meanwhile, the expression of EGF in siEGF group was lower than that in NC group after the transfection (Fig.

A
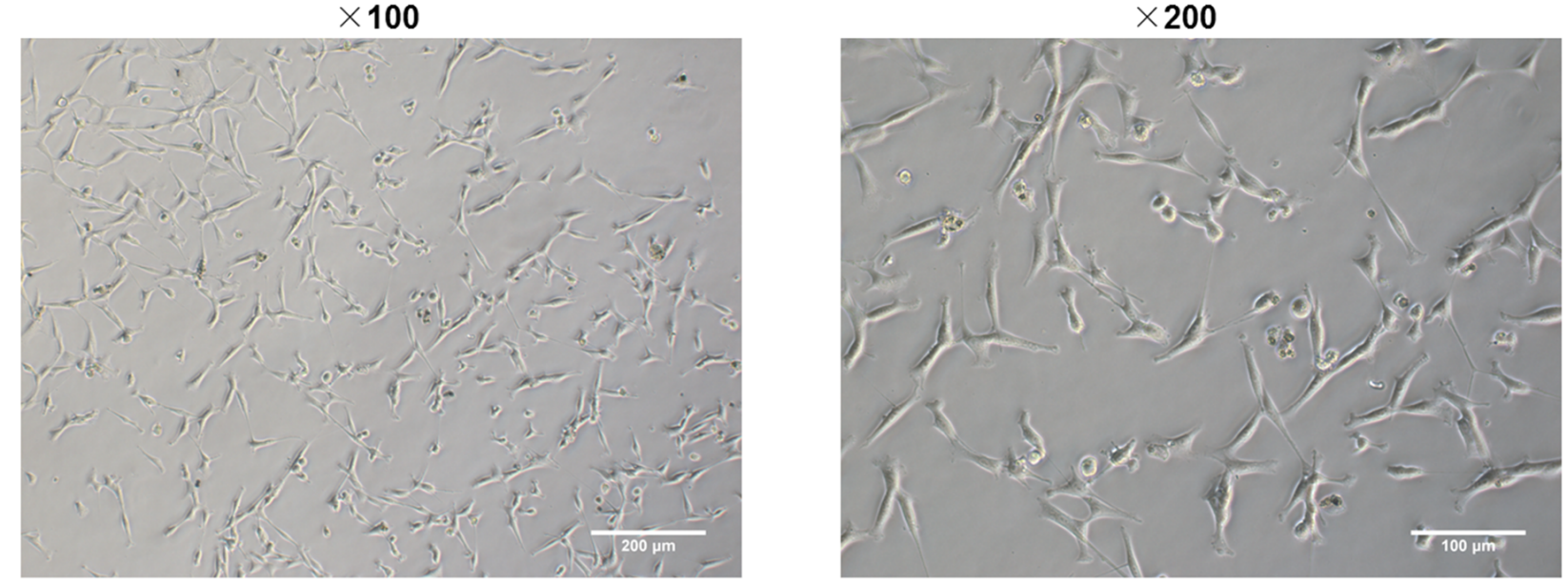

B

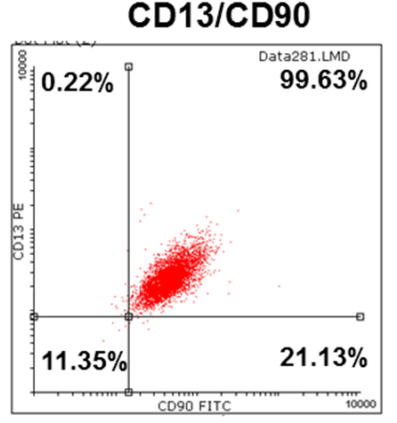

CD14/CD90

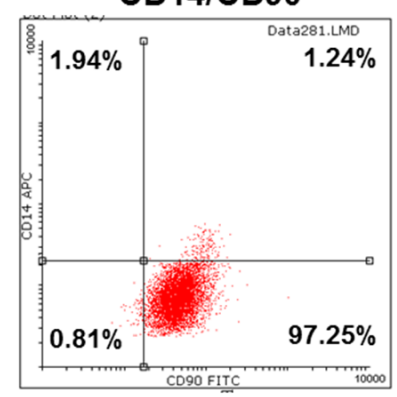

CD44/105

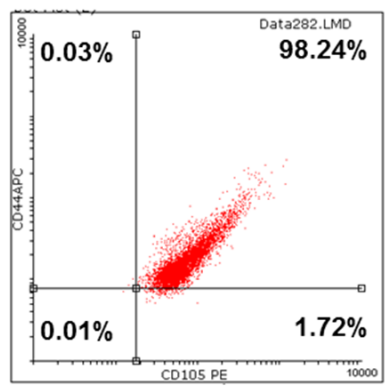

CD44/34

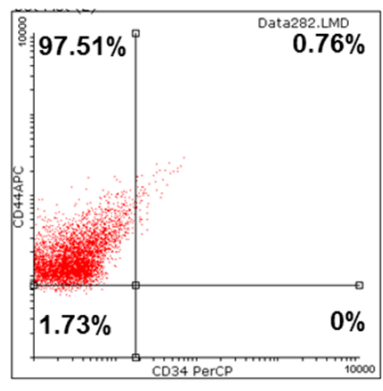

Fig. 1. ADSCs were identified by morphology and immunophenotyping. (A) The representative images of ADSCs $(\times 100,200)$. (B) ADSCs were identified by immunophenotyping, with the detection of CD13, CD14, CD34, CD44, CD90, and CD105. 
$2 \mathrm{~A}$ and $\left.2 \mathrm{~B},{ }^{\star \star} \mathrm{p}<0.01,{ }^{\wedge} \mathrm{p}<0.01\right)$. This effectiveness lasted at least 3 days (Fig. $2 \mathrm{C},{ }^{\star \star}{ }^{\mathrm{p}}<0.01,{ }^{\wedge} \mathrm{p}<0.01$ ), suggesting that EGF could be overexpressed or suppressed constantly in HaCaT cell line.

\section{EGF overexpression upregulated the proliferation of} ADSCs, and its expression regulated the expression of cytokeratin-19 (CK19) and integrin- $\beta$ as well

To investigate whether the expression of EGF could regulate the proliferation of ADSCs and promote the transdifferentiation of ADSCs into epithelial stem cell types, we measured the cell viability of ADSCs by MTT, determined the protein levels of EGF in the bottom chamber and assessed the expressions of CK19 and integrin- $\beta$ in ADSCs. On one hand, the results from the co-cultured ADSCs and HaCaT cells showed that the cell viability in HaCaT-EGF group was higher than that in HaCaT-Mock group, however, no significance between HaCaT-NC and HaCaT-siEGF groups (Fig. $3 \mathrm{~A},{ }^{\star} \mathrm{p}<0.05$ ) was observed. Besides, the protein level of EGF in HaCaT-Mock and HaCaT-NC groups were both higher than that in control group (Fig. $3 \mathrm{~B},{ }^{\star \star} \mathrm{p}<0.01,{ }^{\# \#} \mathrm{p}<0.01,{ }^{\wedge} \mathrm{p}<0.01$ ), indicat- ing that ADSCs and HaCaT cells transfected with lentiviral vector or negative control lentiviral vector alone could produce EGF spontaneously. Moreover, the protein level of EGF in HaCaT-EGF group was higher than that in HaCaT-Mock group, while it was lower in HaCaTsiEGF group than in HaCaT-NC group (Fig. $3 \mathrm{~B},{ }^{\star \star} \mathrm{p}<$ $\left.0.01,{ }^{\# \#} \mathrm{p}<0.01,{ }^{\wedge} \mathrm{p}<0.01\right)$. In addition, the results of the expressions of CK19 and integrin- $\beta$ were consistent with the results of the protein levels of EGF (Fig. $3 \mathrm{C} \sim \mathrm{E},{ }^{\star \star} \mathrm{p}<$ $\left.0.01,{ }^{\# \#} \mathrm{p}<0.01,{ }^{\wedge} \mathrm{p}<0.01\right)$. On the other hand, the results of the culturing of ADSCs with $\mathrm{CM}$ revealed that there were significance between cell viability in EGF-CM group and that in Mock-CM group after 72 hours of culturing, however, unfortunately, no significance between NC-CM and siEGF-CM groups was identified (Fig. 4A, ${ }^{\star} \mathrm{p}<0.05$ ). The outcomes of protein levels of EGF (endogenous and secreted) as well as the expressions of CK19 and integrin- $\beta$ were consistent with the results of the co-culturing of ADSCs and HaCaT cells (Fig. $4 \mathrm{~B} \sim \mathrm{F},{ }^{\star \star} \mathrm{p}<$ $\left.0.01,{ }^{\# \#} \mathrm{p}<0.01,{ }^{\wedge} \mathrm{p}<0.01,{ }^{\#} \mathrm{p}<0.05, \wedge \mathrm{p}<0.05\right)$. Taken together, these results suggested that the proliferation of ADSCs could be elevated by the overexpression of EGF,
A

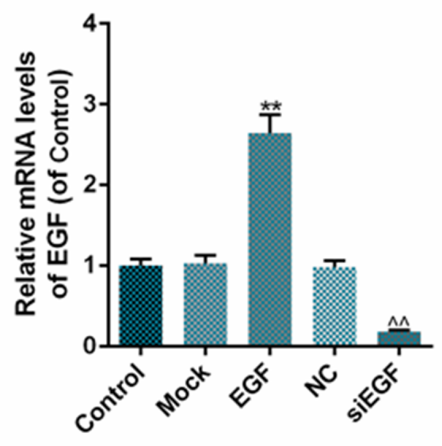

B

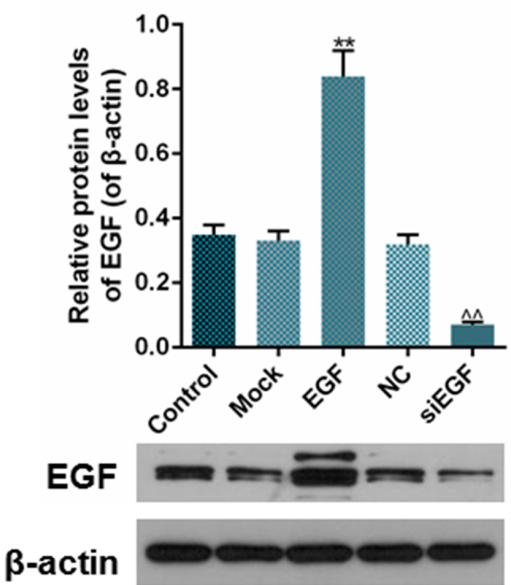

C

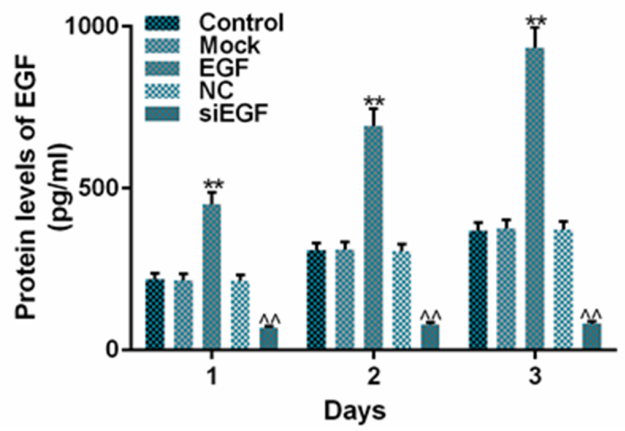

Fig. 2. The expression of EGF after EGF or siEGF transfection into HaCaT cells was detected. (A) Relative mRNA levels of EGF (of control) in each group were detected using RT-qPCR. (B) Relative protein levels of EGF (of $\beta$-actin) in each group were detected using Western blot. (C) Protein levels of excretive EGF (pg/ml) within 3 days in each group were detected using ELISA. Bars indicated mea$\mathrm{ns} \pm \mathrm{SD} .{ }^{* *} \mathrm{p}<0.01$ vs. Mock group; $\wedge^{\wedge}<0.01$ vs. NC group. 
A

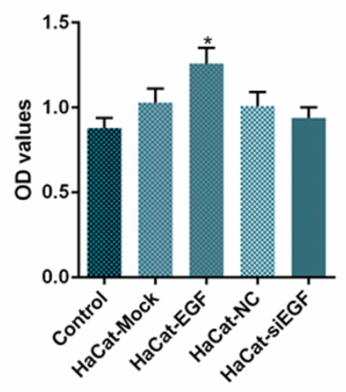

B

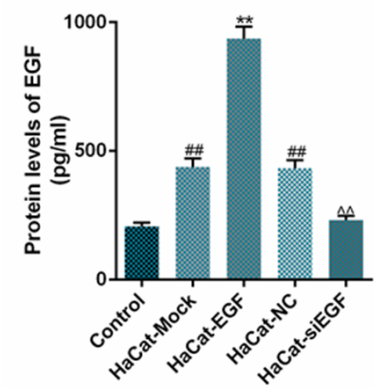

C

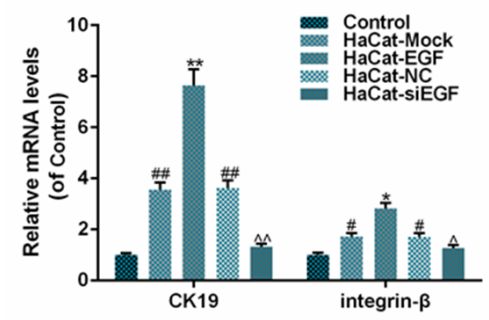

D

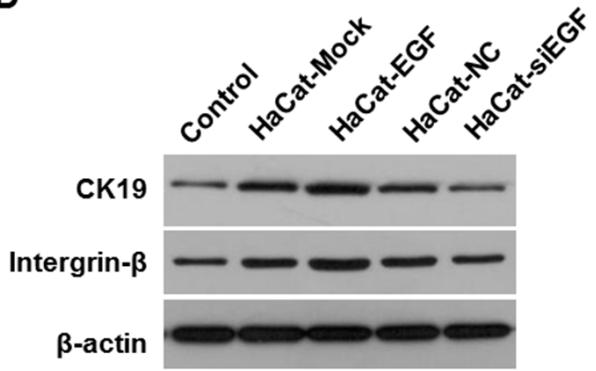

E

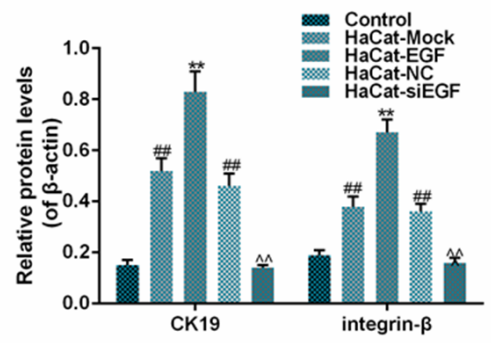

Fig. 3. Effect of EGF expression in HaCat on the viability of ADSCs. (A) Cell viability of ADSCs was detected using MTT assay. (B) Protein levels of excretive EGF (pg/ml) were detected using ELISA. (C) Relative mRNA levels (of control) of CK19 and integrin- $\beta$ were detected using RT-qPCR. (D, E) Relative protein levels (of $\beta$-actin) of CK-19 and integrin- $\beta$ were detected using Western blot. Bars indicated means \pm SD. ${ }^{* *} p<0.01$ and ${ }^{*} p<0.05$ vs. HaCaT-Mock group; ${ }^{\# \#} p<0.01$ and ${ }^{\#} p<0.05$ vs. control group; ${ }^{\wedge} p<0.01$ and ${ }^{\wedge} p<0.05$ vs. HaCat-siEGF group.

A

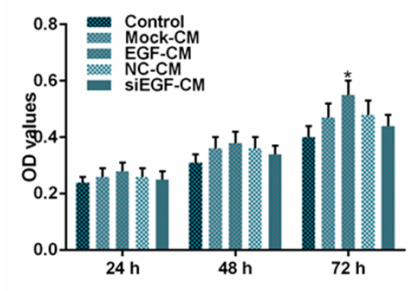

B

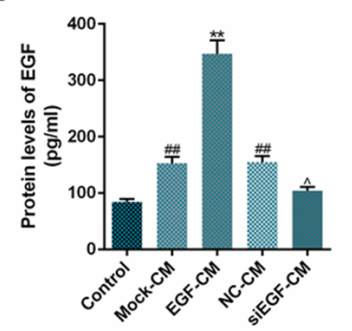

E
C
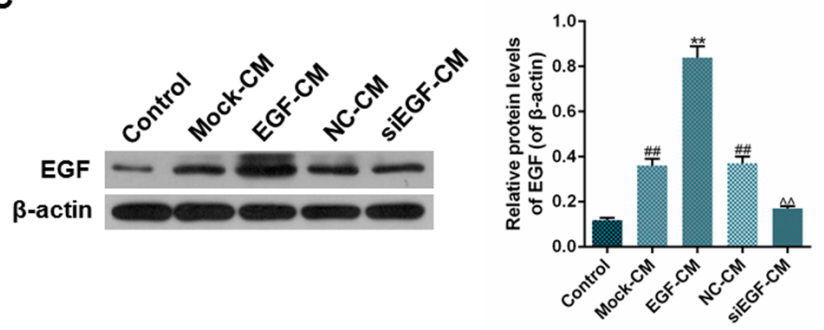

D

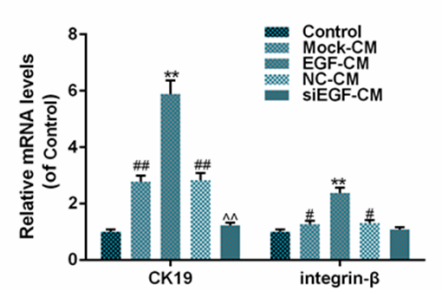

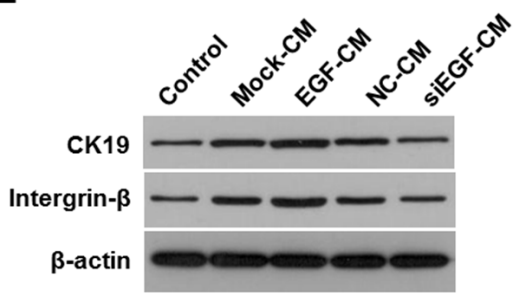

$\mathbf{F}$

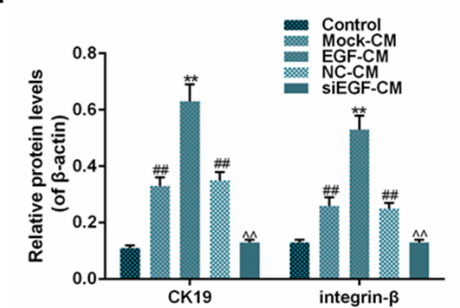

Fig. 4. Effect of EGF-containing medium of EGF-expressed HaCat cells on the viability of ADSCs. (A) Cell viabilities of ADSCs after 24, 48, $72 \mathrm{~h}$ of culturing were detected using MTT assay. (B) Protein levels of excretive EGF (pg/ml) were detected using ELISA. (C) Relative protein levels (of $\beta$-actin) of EGF were detected using Western blot. (D) Relative mRNA levels (of control) of CK19 and integrin- $\beta$ were detected using RT-qPCR. (E, F) Relative protein levels (of $\beta$-actin) of CK-19 and integrin- $\beta$ were detected using Western blot. Bars indicated means \pm SD. ${ }^{* *} p<0.01$ and ${ }^{*} p<0.05$ vs. Mock-CM group; ${ }^{\# \#} p<0.01$ vs. control group; ${ }^{\wedge} p<0.01$ and ${ }^{\wedge} p<0.05$ vs. siEGF-CM group. 


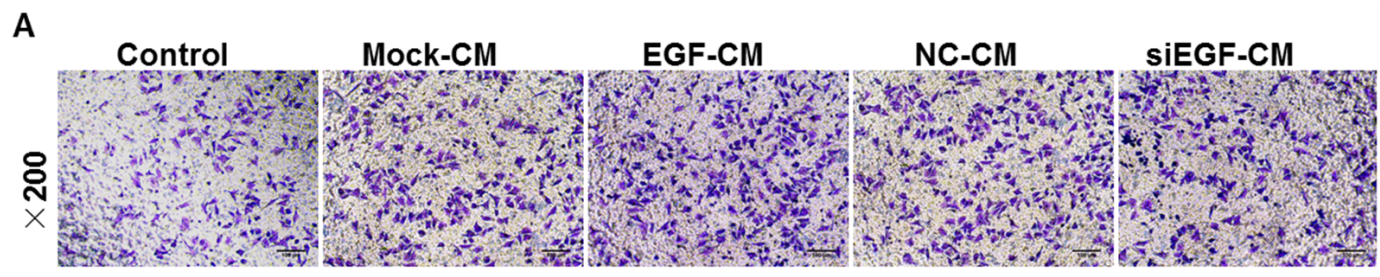

B
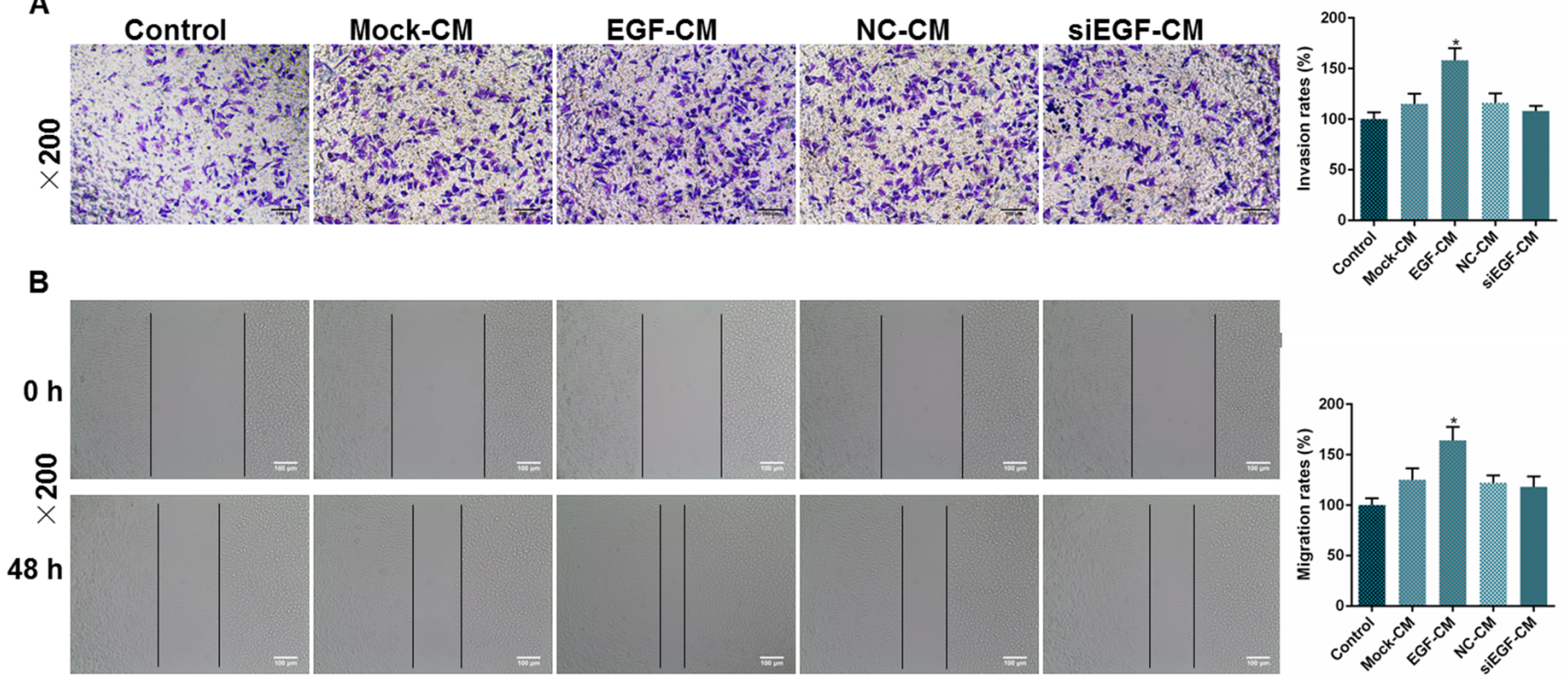

Fig. 5. Effect of EGF-containing medium of EGF-expressed HaCat cells on invasion and migration rates of ADSCs. (A) Cell invasion rates were detected using transwell assay. (B) Cell migration rates were were detected using wound healing assay. Bars indicated means \pm SD. $* \mathrm{p}<0.05$ vs. Mock-CM group.

and the expressions of CK19 and integrin- $\beta$ might be regulated by the expression of EGF.

\section{The overexpression of EGF promoted the migration and invasion rate of ADSCs}

To observe whether the capacity of migration and invasion in ADSCs could be affected by the expression of EGF, we tested the migration and invasion rates of ADSCs by wound healing test and Transwell invasion assay. The results showed that the invasion rate in EGF$\mathrm{CM}$ group was higher than that in Mock-CM group (Fig. $\left.5 \mathrm{~A},{ }^{\star} \mathrm{p}<0.05\right)$. Similarly, the migration rate in EGF-CM group was higher than Mock-CM group (Fig. $5 \mathrm{~B},{ }^{\star} \mathrm{p}<$ $0.05)$. However, no significant difference was observed between NC-CM and siEGF-CM groups on both invasion and migration rates (Fig. 5A and 5B). Thus, we assumed that the overexpression of EGF was able to raise the invasion and migration rates of ADSCs.

\section{Discussion}

In this study, we extracted and identified ADSCs successfully in the first place. Further, after up- and downregulation of the expression of EGF in $\mathrm{HaCaT}$ cells, we explored the alteration of cell viability, migration, invasion and the expressions of CK19 and integrin- $\beta$ by co-culturing ADSCs with modified $\mathrm{HaCaT}$ cells or their CMs. Our study demonstrated the a promising application of EGF in the treatment of skin wound repair using ADSCs.

We first identified the morphology and immunophenotyping of the extracted and cultured cells from adipose tissues. According to the reports, the mature ADSCs would shape-like fibroblast cellsthat have long protruding feet at both ends or are in a flat irregular or polygonal shape with a large protrusion (12). With the extension of cell culture, the fused cells would be arranged as a "swirl" (12). In general, the cells we extracted were as previously described. This evidence, on one hand, confirmed that the cells were ADSCs. Nowadays, it is believed that the positive biomarkers of ADSCs were CD13, CD44, CD90, CD105, while the negative biomarker was CD14 (13). However, the expression of CD34 was still controversial $(13,14)$. Our results showed that the relative expression of CD13, CD44, CD90 and CD105 were high, while CD14 and CD34 were low. On the other hand, we verified the cells were ADSCs to some extent. Taken together, we affirmed that these cells extracted and cultured from adipose tissues were ADSCs, and further experiments could be continued.

HaCaT cells are a type of human immortalized epidermal cell line with the characteristics of epidermal basal cells, which are the same as primary keratinocytes, with stable genetic characteristics but without tumor characteristics (15). HaCaT cell line can replace human normal keratinocytes and is an ideal cell model in the research 
of skin diseases (15). The cell was selected under the consideration that $\mathrm{HaCaT}$ cells being able to continuously express the gene of interest was of great significance in the induction of skin formation in tissue engineering. As expected, we successfully overexpressed and suppressed EGF in $\mathrm{HaCaT}$ cells, and the outcome could last for at least 3 days. Tao et al. (16) demonstrated that HaCaT-EGF cells could be constructed as tissue engineering seed cells by pcDNA3.1-EGF eukaryotic expression vector. Hu et al. (17) suggested that $\mathrm{HaCaT}$ cells modified with human EGF gene could be promising seed cells for building skin substitute. Thus, in this sense, we build a model of EGF-HaCaT seed cells as the basics for further exploration.

Under physiological and pathological conditions, complex interactions between somatic cells as well as between cells and extracellular environment (18) exist. Compared with single-cell culture, the co-culture system can better simulate the environment in vivo and is closer to the somatic characteristics of cells, thus, it is convenient to observe the interactions between cells and cells as well as between cells and extracellular environment (18). Stem cells conditional medium contains a variety of cellular active substances secreted from them, and is easy to be freeze-dried, concentrated, preserved and transported, therefore, it became a research hotspot in the field of regenerative medicine and cell therapy (19-21). By co-culturing modified HaCaT and ADSCs as well as the culturing of ADSCs with CM from modified $\mathrm{HaCaT}$, we revealed that EGF could be able to raise the proliferation of ADSCs, though there was no effect observed after the EGF has been suppressed. Mvula, B. et al. reported that EGF could elevate the proliferation of ADSCs (22). Jia et al. (23) revealed that EGF promoted the proliferation of fibroblasts differentiated from ADSCs. It suggested that EGF could elevate the proliferation of ADSCs. Integrins, a class of glycoprotein receptor family molecules located on the surface of cell membranes, are heterodimers, including $\alpha$ and $\beta$ subunits (24). During the directional differentiation of basal cells, integrin- $\beta$ loses its expression until the cells completely leave the basal layer (24). Thus, integrin- $\beta$ can be used as a surface marker of epidermal like cells $(24,25)$. Keratin is a structural protein of epidermal cells that constitute microfilaments with a diameter of $10 \mathrm{~nm}$ and form a broad network in the cell (26). There are no less than 20 different types of keratin expression in the epithelial tissue of the human body (26). As it has different degrees of differentiation, epidermal cells express different keratin, keratin can also be used as a means of identification of stem cells, committed progen- itor cells and differentiated cells (26). Epidermal like cell expresses CK19, hence it can be seen as markers for epidermal stem cells (26). Our results revealed that the expressions of CK19 and integrin- $\beta$ were elevated using the method of co-culturing modified $\mathrm{HaCaT}$ and ADSCs and the culturing of ADSCs with CM from modified HaCaT. Early in 1999, a report showed that the function of integrin- $\beta 1$ could be stimulated by EGF in metastatic breast carcinoma cell line MDA-MB-435 (27). Moreover, Yoneda et al. (28) reported that EGF induced CK19 expression with increased growth abilities in human hepatocellular carcinoma. Makarova et al. (29) demonstrated a dose-dependent increase of CK19 expression after EGF treatment in UT-SCC-26A cells. Therefore, we speculated that the expression of EGF could regulate the expressions of CK19 and integrin- $\beta$, showing the regulatory role in the induction of transdifferentiating ADSCs into epidermal like cells.

Our results showed that the invasion and migration rates of ADSCs could be elevated by the culturing of ADSCs with CM from modified HaCaT. However, the $\mathrm{CM}$ of HaCaT with suppressed expression of EGF did not show significant effect on the invasion or migration of ADSCs. However, there might be a limitation not inhibiting proliferation ability when studying migration ability, which would be further studied. Recently, Chen et al. (30) reported that EGF released from human platelets promoted the invasion, migrationand epithelial mesenchymal transition (EMT) of oral squamous cell carcinoma. Tumur et al. (31) showed that EGF stimulated the migration and invasion of head and neck squamous cell carcinoma. Thus, it suggested that the overexpression of EGF could elevate the migration and invasion rates of ADSCs, which would accelerate the healing of skin wound. However, further study is required to determine the role of suppressed EGF.

In conclusion, EGF overexpressed $\mathrm{HaCaT}$ cells could be served as a stimulus to promote the proliferation, migration, and invasion and the transdifferentiation into epidermal stem cell immunophenotyping of ADSCs. Our findings suggested that EGF has a promising effect on the repair of skin wound. It provided evidence for $\mathrm{HaCaT}$ cells becoming mother-cells in the tissue engineering. The deeper mechanism would be studied in the future, including the expression of EGFR and EGF related signals etc.

\section{Acknowledgments}

This work was supported by Project of Guangdong Sceience and Technology Department [Grant number: 2013B0218002]. 


\section{Potential Conflict of Interest}

The authors have no conflicting financial interest.

\section{Author Contributions}

Substantial contributions to conception and design: YM, JM. Data acquisition, data analysis and interpretation: YX, XX. Drafting the article or critically revising it for important intellectual content: YM, JM, XX. Final approval of the version to be published: All authors. Agreement to be accountable for all aspects of the work in ensuring that questions related to the accuracy or integrity of the work are appropriately investigated and resolved: YX, XX.

\section{References}

1. Inzucchi SE, Bergenstal RM, Buse JB, Diamant $M$, Ferrannini E, Nauck M, Peters AL, Tsapas A, Wender R, Matthews DR. Management of hyperglycaemia in type 2 diabetes, 2015: a patient-centred approach. Update to a position statement of the American Diabetes Association and the European Association for the study of diabetes. Diabetologia 2015;58:429-442

2. Takeo $M$, Lee W, Ito $M$. Wound healing and skin regeneration. Cold Spring Harb Perspect Med 2015;5:a023267

3. Sharma RR, Pollock K, Hubel A, McKenna D. Mesenchymal stem or stromal cells: a review of clinical applications and manufacturing practices. Transfusion 2014;54:14181437

4. Skubis A, Gola J, Sikora B, Hybiak J, Paul-Samojedny M, Mazurek U, Łos MJ. Impact of antibiotics on the proliferation and differentiation of human adipose-derived mesenchymal stem cells. Int J Mol Sci 2017;18:E2522

5. Naderi N, Combellack EJ, Griffin M, Sedaghati T, Javed M, Findlay MW, Wallace CG, Mosahebi A, Butler PE, Seifalian AM, Whitaker IS. The regenerative role of adipose-derived stem cells (ADSC) in plastic and reconstructive surgery. Int Wound J 2017;14:112-124

6. Brzoska M, Geiger H, Gauer S, Baer P. Epithelial differentiation of human adipose tissue-derived adult stem cells. Biochem Biophys Res Commun 2005;330:142-150

7. Yao YM, Yan H, Zhang ZM, Wu CF, Zhang L, Yang BB. [Effects of rabbit adipose-derived mesenchymal stem cells on the healing of skin deep partial-thickness scald wound of rabbit]. Zhonghua Shao Shang Za Zhi 2016;32:402-407. Chinese

8. Swindle CS, Tran KT, Johnson TD, Banerjee P, Mayes AM, Griffith L, Wells A. Epidermal growth factor (EGF)-like repeats of human tenascin-C as ligands for EGF receptor. J Cell Biol 2001;154:459-468

9. Alexandrescu DT, Kauffman CL, Dasanu CA. The cutaneous epidermal growth factor network: can it be translated clinically to stimulate hair growth? Dermatol Online J 2009;15:1

10. Long JL, Zuk P, Berke GS, Chhetri DK. Epithelial differ- entiation of adipose-derived stem cells for laryngeal tissue engineering. Laryngoscope 2010;120:125-131

11. Li H, Xu Y, Fu Q, Li C. Effects of multiple agents on epithelial differentiation of rabbit adipose-derived stem cells in 3D culture. Tissue Eng Part A 2012;18:1760-1770

12. Fève B. Adipogenesis: cellular and molecular aspects. Best Pract Res Clin Endocrinol Metab 2005;19:483-499

13. Bourin P, Bunnell BA, Casteilla L, Dominici M, Katz AJ, March KL, Redl H, Rubin JP, Yoshimura K, Gimble JM. Stromal cells from the adipose tissue-derived stromal vascular fraction and culture expanded adipose tissue-derived stromal/stem cells: a joint statement of the International Federation for Adipose Therapeutics and Science (IFATS) and the International Society for Cellular Therapy (ISCT). Cytotherapy 2013;15:641-648

14. Jiang A, Li M, Duan W, Dong Y, Wang Y. Improvement of the survival of human autologous fat transplantation by adipose-derived stem-cells-assisted lipotransfer combined with bFGF. ScientificWorldJournal 2015;2015:968057

15. Schoop VM, Mirancea N, Fusenig NE. Epidermal organization and differentiation of $\mathrm{HaCaT}$ keratinocytes in organotypic coculture with human dermal fibroblasts. J Invest Dermatol 1999;112:343-353

16. Tao K, Bai XZ, Zhang ZF, Shi JH, Hu XL, Tang CW, Hu $\mathrm{DH}$, Han JT. Construction of the tissue engineering seed cell (HaCaT-EGF) and analysis of its biological characteristics. Asian Pac J Trop Med 2013;6:893-896

17. Hu DH, Zhang ZF, Zhang YG, Zhang WF, Wang HT, Cai WX, Bai XZ, Zhu HY, Shi JH, Tang CW. A potential skin substitute constructed with hEGF gene modified HaCaT cells for treatment of burn wounds in a rat model. Burns 2012;38:702-712

18. Paschos NK, Brown WE, Eswaramoorthy R, Hu JC, Athanasiou KA. Advances in tissue engineering through stem cell-based co-culture. J Tissue Eng Regen Med 2015;9: 488-503

19. Pawitan JA. Prospect of stem cell conditioned medium in regenerative medicine. Biomed Res Int 2014;2014:965849

20. Sugitani S, Tsuruma K, Ohno Y, Kuse Y, Yamauchi M, Egashira Y, Yoshimura S, Shimazawa M, Iwama T, Hara H. The potential neuroprotective effect of human adipose stem cells conditioned medium against light-induced retinal damage. Exp Eye Res 2013;116:254-264

21. Satarian L, Javan M, Kiani S, Hajikaram M, MirnajafiZadeh J, Baharvand H. Engrafted human induced pluripotent stem cell-derived anterior specified neural progenitors protect the rat crushed optic nerve. PLoS One 2013;8: e71855

22. Mvula B, Moore TJ, Abrahamse H. Effect of low-level laser irradiation and epidermal growth factor on adult human adipose-derived stem cells. Lasers Med Sci 2010;25:33-39

23. Jia YY, Zhou JY, Chang Y, An F, Li XW, Xu XY, Sun XL, Xiong CY, Wang JL. Effect of optimized concentrations of basic fibroblast growth factor and epidermal growth factor on proliferation of fibroblasts and expression of collagen: related to pelvic floor tissue regeneration. Chin Med J 
(Engl) 2018;131:2089-2096

24. Suzuki Y, Yanagisawa M, Yagi H, Nakatani Y, Yu RK. Involvement of betal-integrin up-regulation in basic fibroblast growth factor- and epidermal growth factor-induced proliferation of mouse neuroepithelial cells. J Biol Chem 2010;285:18443-18451

25. Watt FM. Epidermal stem cells: markers, patterning and the control of stem cell fate. Philos Trans R Soc Lond B Biol Sci 1998;353:831-837

26. Michel M, Török N, Godbout MJ, Lussier M, Gaudreau P, Royal A, Germain L. Keratin 19 as a biochemical marker of skin stem cells in vivo and in vitro: keratin 19 expressing cells are differentially localized in function of anatomic sites, and their number varies with donor age and culture stage. J Cell Sci 1996;109(Pt 5):1017-1028

27. Adelsman MA, McCarthy JB, Shimizu Y. Stimulation of betal-integrin function by epidermal growth factor and heregulin-beta has distinct requirements for erbB2 but a similar dependence on phosphoinositide 3-OH kinase. Mol Biol Cell 1999;10:2861-2878

28. Yoneda N, Sato Y, Kitao A, Ikeda H, Sawada-Kitamura S,
Miyakoshi M, Harada K, Sasaki M, Matsui O, Nakanuma Y. Epidermal growth factor induces cytokeratin $19 \mathrm{ex}-$ pression accompanied by increased growth abilities in human hepatocellular carcinoma. Lab Invest 2011;91:262-272

29. Makarova G, Bette M, Schmidt A, Jacob R, Cai C, Rodepeter F, Betz T, Sitterberg J, Bakowsky U, Moll R, Neff A, Sesterhenn A, Teymoortash A, Ocker M, Werner JA, Mandic R. Epidermal growth factor-induced modulation of cytokeratin expression levels influences the morphological phenotype of head and neck squamous cell carcinoma cells. Cell Tissue Res 2013;351:59-72

30. Chen R, Jin G, Li W, McIntyre TM. Epidermal Growth Factor (EGF) autocrine activation of human platelets promotes EGF receptor-dependent oral squamous cell carcinoma invasion, migration, and epithelial mesenchymal transition. J Immunol 2018;201:2154-2164

31. Tumur Z, Katebzadeh S, Guerra C, Bhushan L, Alkam T, Henson BS. RhoC mediates epidermal growth factor-stimulated migration and invasion in head and neck squamous cell carcinoma. Neoplasia 2015;17:141-151 